$6-1-2020$

\title{
Comparing Means under Heteroscedasticity and Nonnormality: Further Exploring Robust Means Modeling
}

\author{
Alyssa Counsell \\ York University, Toronto \\ Robert Philip Chalmers \\ York University, Toronto \\ Robert A. Cribbie \\ York University, Toronto, cribbie@yorku.ca
}

Follow this and additional works at: https://digitalcommons.wayne.edu/jmasm

Part of the Applied Statistics Commons, Social and Behavioral Sciences Commons, and the Statistical Theory Commons

\section{Recommended Citation}

Counsell, Alyssa; Chalmers, Robert Philip; and Cribbie, Robert A. (2020) "Comparing Means under Heteroscedasticity and Nonnormality: Further Exploring Robust Means Modeling," Journal of Modern Applied Statistical Methods: Vol. 18 : Iss. 1 , Article 28.

DOI: $10.22237 / \mathrm{jmasm} / 1571659200$

Available at: https://digitalcommons.wayne.edu/jmasm/vol18/iss1/28

This Regular Article is brought to you for free and open access by the Open Access Journals at DigitalCommons@WayneState. It has been accepted for inclusion in Journal of Modern Applied Statistical Methods by an authorized editor of DigitalCommons@WayneState. 


\section{Comparing Means under Heteroscedasticity and Nonnormality: Further Exploring Robust Means Modeling}

\author{
Alyssa Counsell \\ Ryerson University \\ Toronto, Ontario, Canada
}

\author{
Robert Philip Chalmers \\ York University \\ Toronto, Ontario, Canada
}

\author{
Robert A. Cribbie \\ York University \\ Toronto, Ontario, Canada
}

Comparing the means of independent groups is a concern when the assumptions of normality and variance homogeneity are violated. Robust means modeling (RMM) was proposed as an alternative to ANOVA-type procedures when the assumptions of normality and variance homogeneity are violated. The purpose of this study is to compare the Type I error and power rates of RMM to the trimmed Welch procedure. A Monte Carlo study was used to investigate RMM and the trimmed Welch procedure under several conditions of nonnormality and variance heterogeneity. The results suggest that the trimmed Welch provides a better balance of Type I error control and power than RMM.

Keywords: robust means modeling, Yuen test, trimmed means, nonnormality, heteroscedasticity

The independent samples analysis of variance (ANOVA) is a popular statistical analysis in psychology because it is common to examine mean differences across multiple groups. Certain assumptions must be met to validly interpret the results of the ANOVA, which are: independence of observations, normality of population distributions, and equal population variances. Although the assumption of independence is an issue at the research design level, normality and equal variance are important statistical assumptions that should be examined when conducting the ANOVA.

These assumptions are rarely satisfied with the types of data typically collected within psychology and other social science fields (Blanca, Arnau, Lopez-Montiel, Bono, \& Bendayan, 2011; Golinski \& Cribbie, 2009; Keselman et al., 1998, Micceri, 1989; Wilcox, 1990a, 1990b). The Type I error rates and power of the ANOVA are typical research data that are affected by violating assumptions (e.g., Boneau, 1960; Glass, Peckham, \& Sanders, 1972), and frequently these assumptions are ignored (Hoekstra, Kiers, \& Johnson, 2012). Nevertheless, ANOVA continues to be used 


\section{ALYSSA COUNSELL ET AL.}

despite the availability of improved methods for comparing central tendencies under these circumstances (e.g., Cribbie, Fiksenbaum, Wilcox, \& Keselman, 2012; Keselman, Algina, Lix, Wilcox, 1995, 2017; Keselman, Algina, Wilcox, \& Deering, 2008).

Assumption violation may result in inaccurate interpretations of true population differences for the traditional ANOVA procedure. For example, when one population's variance is much higher than another, especially with unequal sample sizes, the empirical probability of Type I errors deviates from the nominal level (Box, 1954, Brown \& Forsythe, 1974a, 1974b; Wilcox, 1988). The manner in which Type I error rates are affected depends on the pairing of sample size and variance heterogeneity. Specifically, when small sample sizes are paired with large variance (i.e., negative/ inverse pairing), empirical Type I error rates will be inflated relative to the nominal $\alpha$, whereas when large sample sizes are paired with large variance (i.e., positive/ direct pairing), Type I error rates tend to be too conservative. In instances where the homogeneity of variance assumption has been met, deviations from normality tend to have little effect on the Type I error rates of the traditional ANOVA, but often decrease the statistical power (Harwell, Rubinstein, Hayes, \& Olds, 1992; Lix, Keselman, \& Keselman, 1996). When population variances are unequal and distributions are nonnormal, empirical Type I error rates are extremely aberrant (Cribbie et al., 2012).

\section{Trimmed Welch Test with Winsorized Variances}

Due to the routine violation of assumptions in psychological research, many researchers have proposed alternatives to the omnibus ANOVA $F$ test. One method to maintain accurate Type I error control and retain power under variance heterogeneity and nonnormality is to use trimmed means and Winsorized variances in combination with a test that uses a non-pooled standard error and adjusted degrees of freedom (trimmed Welch; e.g., Cribbie et al., 2012; Keselman, Kowalchuk, \& Lix, 1998; Keselman, Algina, Wilcox, \& Kowalchuk, 2000; Keselman et al., 2008; Wilcox, Keselman, Muska, \& Cribbie, 2000). The trimmed Welch test performs well even with extremely nonnormal distributions and disparate sample sizes and variances. Details on this test statistic are given below.

Let the effective sample size (i.e., the sample size after trimming), be $h=N-2 \lambda$ where $\lambda=[\kappa n]$, where $\kappa$ is the proportion of trimming from each tail and $[\kappa n]$ is the largest integer $\leq \kappa n$. Then, the sample trimmed mean is:

$$
\bar{X}_{t}=\frac{1}{h} \sum_{i=\lambda+1}^{n-\lambda} X_{i}
$$


The sample Winsorized mean is:

$$
\bar{X}_{W}=\frac{1}{n} \sum_{i}^{N} Y_{i}
$$

where

$$
Y_{i}=\left\{\begin{array}{l}
X_{(\lambda+1)} \text { if } X_{i} \leq X_{(\lambda+1)} \\
X_{i} \text { if } X_{(\lambda+1)}<X_{i}<X_{(n-\lambda)} \\
X_{(n-\lambda)} \text { if } X_{i} \geq X_{(n-\lambda)}
\end{array}\right.
$$

The sample Winsorized variance is:

$$
s_{W}^{2}=\frac{\sum_{i}\left(Y_{i}-\bar{X}_{W}\right)^{2}}{n-1}
$$

Let $n_{j}, h_{j}, s_{W j}$, and $\bar{X}_{\mathrm{tj}}$ represent the values of $n, h, s_{W}$, and $\bar{X}_{\mathrm{t}}$ for the $j$ th group, and

$$
\begin{gathered}
q_{j}=\frac{\left(n_{j}-1\right) s_{w_{j}}^{2}}{h_{j}\left(h_{j}-1\right)}, \\
w_{j}=\frac{1}{q_{j}}, \\
U=\sum_{j} w_{j}, \\
\tilde{X}=\frac{1}{U} \sum_{i} w_{j} \bar{X}_{t j}, \\
A=\frac{1}{J-1} \sum_{j} w_{j}\left(\bar{X}_{t j}-\tilde{X}\right)^{2}, \\
B=\frac{2(J-2)}{J^{2}-1} \sum_{j} \frac{\left(1-\frac{w_{j}}{U}\right)^{2}}{h_{j}-1}, \text { and } \\
F_{t}=\frac{A}{B+1}
\end{gathered}
$$

The null hypothesis when using sample trimmed means is $\mathrm{H}_{\mathrm{o}}: \mu_{\mathrm{t} 1}=\ldots=\mu_{\mathrm{t}}$ (i.e., the population trimmed means are equal), and is rejected if $F_{t} \geq F_{a, \mathrm{~J}-1}, v_{\mathrm{W}}$, where: 


$$
v_{\mathrm{Wt}}=\frac{1}{\frac{3}{J^{2}-1} \sum_{j} \frac{\left(1-\frac{w_{j}}{U}\right)^{2}}{h_{j}-1}}
$$

Fan and Hancock (2012) noted criticisms with using a non-pooled standard error test in combination with trimming extreme observations. Specifically, there should be hesitation using trimming because it involves temporarily removing a portion of the data. The null hypothesis relates to trimmed, not ordinary, population mean differences. Also, the Type I error rates and power of these techniques may not be satisfactory with larger degrees of nonnormality. The first two criticisms hold if the interest is only in comparing the full distributions of the populations, however if the interest is in comparing the bulk of the distributions, and limiting the effects of outliers or heavy tailed distributions, then these criticisms do not hold. Moving from a traditional null hypothesis (e.g., $\mathrm{H}_{0}: \mu_{1}=\mu_{2}$ ) to a robust null hypothesis (e.g., $\mathrm{H}_{0}$ : $\mu_{\mathrm{t} 1}=\mu_{\mathrm{t} 2}$, where $\mu_{\mathrm{t}}$ represents the trimmed population mean) has little effect on the overall testing strategy (i.e., it simply eliminates the extreme scores from the analysis). It is typically preferred when the outlying cases have undue influence on the results of the analyses. With regard to the final criticism, the trimmed Welch has been found to be superior to alternative procedures when distributions are nonnormal and population variances are unequal (Cribbie et al., 2012). These potential limitations led Fan and Hancock to propose a new structural equation modeling (SEM; Bollen, 1989) based approach entitled robust means modeling.

\section{Robust Means Modeling}

Robust means modeling (RMM; Fan \& Hancock, 2012) is a SEM technique inspired by Sorbom's (1974) structured means modeling (SMM). It is a special case of SMM where the means being compared are observed variables (e.g., an ANOVA model) instead of latent variables (Fan \& Hancock, 2012). The SMM approach can be represented in matrix form by the following model:

$$
\boldsymbol{x}=\boldsymbol{v}_{\boldsymbol{k}}+\Lambda_{k} \xi+\delta
$$

where $\boldsymbol{x}$ is a $p \times 1$ vector of observed indicators of a latent variable, $\boldsymbol{\xi}$; $v_{k}$ is a $p \times 1$ vector of intercepts; $\boldsymbol{\Lambda}_{k}$ is a $p \times 1$ vector of factor loadings $\lambda$; and $\boldsymbol{\delta}$ is a $p \times 1$ vector of errors.

The model for RMM is a simpler version of the SMM model, because there are no latent variables $(\boldsymbol{\xi})$ and therefore no factor loadings $\left(\boldsymbol{\Lambda}_{k}\right)$ leaving only: $x=v_{k}+\delta$ 


\section{FURTHER EXPLORING ROBUST MEANS MODELING}

(Fan \& Hancock, 2012). The null hypothesis remains $\mathrm{H}_{0}: v_{1}=v_{2}=\ldots=v_{K}$, where $v$ represents the population intercepts/means, but the method for comparing the means differs. Specifically, the means are constrained to be equal in the SEM model and the variances are free to be estimated, thereby removing the homogeneity of variance assumption. The SMM model can be estimated through a weighted combination of the multi-group maximum likelihood (ML) fit functions:

$$
F_{M L}=\sum_{k=1}^{K}\left(\frac{n_{k}}{N}\right) F_{k}\left(\boldsymbol{S}_{k}, \boldsymbol{m}_{k}, \hat{\Sigma}_{k}, \hat{\boldsymbol{\mu}}_{k}\right)
$$

where $n_{k}$ is the sample size of the $k$ th group, $N$ is the total sample size for all groups, $\boldsymbol{S}_{k}$ is the $k$ th group's observed covariance matrix, $\boldsymbol{m}_{k}$ is the $k$ th group's observed mean vector, $\hat{\Sigma}_{k}$ is the $k$ th group's model-implied covariance matrix, $\hat{\boldsymbol{\mu}}_{k}$ is the $k$ th group's model-implied vector of means and $F_{k}$ is the $k$ th group's ML fit function defined as:

$$
F_{k}=\left[\ln \left|\hat{\Sigma}_{k}\right|+\operatorname{tr}\left(\boldsymbol{S}_{k} \hat{\Sigma}_{k}^{-1}\right)-\ln \left|\boldsymbol{S}_{k}\right|-p\right]+\left(\boldsymbol{m}_{k}-\hat{\boldsymbol{\mu}}_{k}\right)^{\prime} \hat{\sum}_{k}^{-1}\left(\boldsymbol{m}_{k}-\hat{\boldsymbol{\mu}}_{k}\right)
$$

where $p$ is the number of observed variables (i.e., 1 for RMM).

$F_{M L}$ can be used to calculate a test statistic that quantifies evidence against the null hypothesis of mean equality. Specifically $T_{M L}=(N-1) F_{M L}$ with degrees of freedom $(d f)=K p(p+3) / 2-q$, where $K$ is the number of groups, $p$ is the number of observed variables, and $q$ is the number of parameters estimated for the model (Fan \& Hancock, 2012). The only parameters estimated in the RMM model are the $K$ population variances plus one population mean (constrained to be equal across the $K$ groups). Therefore, the $d f$ in the RMM model simplifies considerably to $K-1 . T_{M L}$ follows a $\chi^{2}$ distribution when data are conditionally normal, but it becomes biased as data become less normally distributed.

Although traditional ML estimation requires conditional multivariate normality to produce unbiased results, there are a number of modified estimation procedures designed to alleviate issues stemming from nonnormality (e.g., Browne, 1984; Satorra $\&$ Bentler, 2001; Yuan \& Bentler, 1999). The aim of this study is to test many of the original RMM procedures in Fan and Hancock's (2012).

\section{Asymptotically distribution free (ADF) method}

One of the first modifications to ML is Browne's (1984) ADF method. It is also known as arbitrary generalized least squares (AGLS) or weighted least squares (WLS). Unlike traditional ML, the ADF method does not require the multivariate normality assumption as a condition for its use. The ADF method is based on the 


\section{ALYSSA COUNSELL ET AL.}

generalized least squares approach, but uses a different weight matrix that allows for nonnormal data. It can be written as the following weighted fit function for multiple groups:

$$
F_{A D F}=\sum_{k=1}^{K}\left(\boldsymbol{s}_{k}-\hat{\boldsymbol{\sigma}}_{k}\right)^{\prime} W_{k}^{-1}\left(\boldsymbol{s}_{k}-\hat{\boldsymbol{\sigma}}_{k}\right)
$$

where $s_{k}$ is the $p^{*} \mathrm{x} 1$ vector of first and second moments of the distribution of observed means, variances, and covariances $\left(p^{*}=p(p+3) / 2\right), \hat{\sigma}_{k}$ is the $p^{*} \times 1$ vector of model-implied first and second moments, and $W_{k}^{-1}$ is an inverted $p^{*} \times p^{*}$ weight matrix of higher moments. For more details about the weight matrix see Browne (1984) or Muthén (1989). Using the ADF method, obtain the test statistic

$$
T_{A D F}=(N-1) F_{A D F}
$$

which is distributed as $\chi^{2}$ with $K-1 d f$. In theory, the ADF method solves estimation issues arising from models with nonnormal data, but simulation studies demonstrate that it requires very large sample sizes and may be limited in the number of variables in the SEM model to obtain stable estimates for the weight matrix (e.g., Curran, West, \& Finch, 1996; Finch, West, and MacKinnon, 1997; Muthén, \& Kaplan, 1992; Olsson, Foss, Troye, \& Howell, 2000).

Modified ADF methods. Given the issues discussed above concerning the ADF method, modifications were proposed to correct for estimation issues resulting from small sample sizes. For example, Fan and Hancock (2012) recommended two modified ADF methods by Yuan and Bentler $(1997 ; 1999)$. The first statistic (YB1; Yuan \& Bentler, 1997) modifies the ADF statistic as follows:

$$
T_{Y B 1}=\frac{T_{A D F}}{\left(1+T_{A D F} N^{-1}\right)}
$$

The $T_{Y B 1}$ follows a $\chi^{2}$ distribution and has the same $d f$ as the ADF model ( $K-1$ for the RMM model).

Yuan and Bentler's (1999) second modified ADF statistic (YB2) follows an $F$ distribution and can be expressed by the following equation:

$$
T_{Y B 2}=T_{A D F} \frac{\left(N-\left(K p^{*}-q\right)\right)}{(N-1)\left(K p^{*}-q\right)}
$$




\section{FURTHER EXPLORING ROBUST MEANS MODELING}

with numerator $d f=K p^{*}-q$ and denominator $d f=N-\left(K p^{*}-q\right)$. In RMM, the $d f$ simplifies to $K-1$ for the numerator and $N-K+1$ for the denominator $d f$ (Fan \& Hancock).

Scaling corrections to ML. The Satorra-Bentler (SB; Satorra \& Bentler, 1988) rescaled test statistic is another popular alternative to traditional ML estimation, which was extended to include mean testing (Satorra, 1992). The new statistic, $T_{S B}=T_{M L} \hat{c}^{-1}$ where $\hat{c}^{-1}$ is a scaling factor that takes into account the model, estimation procedure, and degree of kurtosis. It is approximately distributed as $\chi^{2}$ with the same $d f$ as $T_{M L}$ and includes the use of robust standard errors. For technical details about the scaling constant see Satorra (1992) or Satorra and Bentler (2001). The SB rescaled test has been found to perform well in general, and better than the ADF methods with smaller sample sizes (e.g., Hu, Bentler, \& Kano, 1992; Curran, West, \& Finch, 1996). Given these options, RMM is a promising method as it includes robust estimation techniques to combat issues with nonnormal data and allows for distinct model estimates of population variances.

\section{Performance of the RMM Methods}

Fan and Hancock (2012) evaluated the performance of several RMM procedures in comparison to four modified ANOVA procedures along with the traditional ANOVA $F$-test as a reference. The alternative ANOVA procedures included the Welch test (Welch, 1951), Brown and Forsythe method (Brown \& Forsythe, 1974c), AlexanderGovern method (Alexander \& Govern, 1994), and James' second order test (James, 1951). In Fan and Hancock's study, trimmed means and Winsorized variances were incorporated into each of the four ANOVA alternatives. Under various conditions of nonnormality and unequal population variances, they found the RMM procedures outperformed the traditional methods and alternatives with regard to Type I error rates and power when moderate to extreme amounts of nonnormality were combined with unequal sample sizes and variances. They reported liberal Type I error rates for the modified ANOVA tests including the trimmed Welch, and due to inaccurate Type I error rates, power results were not reported. It was, however, noted the power of the RMM methods was higher than the ANOVA-based methods, although the power difference between the approaches decreased as sample size increased.

Fan and Hancock's (2012) results contrasted with previous research demonstrating that the Welch test with trimmed means and Winsorized variances has accurate Type I error rates and adequate power results (e.g., Cribbie et al, 2012; Lix et al., 1996). Fan and Hancock found slight differences in performance across the RMM methods, depending on the condition, but overall the pattern of results was similar 


\section{ALYSSA COUNSELL ET AL.}

for the procedures. For Type I error rates, the RMM methods all provided good results, only deviating substantially from the nominal level in a few conditions. The results were significantly better than the ANOVA-based methods. The RMM method with the highest power was Browne's (1984) ADF method, followed by the two Yuan and Bentler $(1997 ; 1999)$ statistics. Based on the overall performance of all of the methods under study, Fan and Hancock recommended the two Yuan and Bentler adjusted ADF methods over the ANOVA-based methods and other RMM approaches.

\section{Study Objectives}

Given the promising results for RMM procedures, the intent here is to extend the findings of Fan and Hancock (2012) in two ways. First, data were simulated from two different families of nonnormal distributions not investigated by Fan and Hancockthe $g$ and $h$ distribution (Hoaglin, 1985) and the $\chi^{2}$ distribution. Results on the performance of the RMM procedures are included when the distribution shapes differed across groups (e.g., one group had positively skewed data, another group had normally distributed or negatively skewed data, etc.). As in Fan and Hancock (2012), the trimmed Welch is included, because it is widely recommended for comparing population means under nonnormality and variance heterogeneity (Cribbie et al., 2012; Wilcox, 2017). The poor performance of the trimmed Welch in Fan and Hancock was unexpected and deserves further investigation. The expanded conditions of the current paper will allow further comparisons between the trimmed Welch and the RMM procedures.

\section{Methodology}

A Monte Carlo study was constructed to evaluate the performance (i.e., power and Type I error rates) of traditional ANOVA-based methods and RMM tests for comparing independent group means across many conditions of nonnormality and variance heterogeneity. The ANOVA-based methods included the traditional ANOVA (for baseline comparisons only), Welch's (1951) heteroscedastic procedure with both usual means and variances (Welch) and trimmed means (20\% symmetric trimming) and Winsorized variances (T Welch). The RMM methods included the traditional maximum-likelihood (ML) approach based on a $\chi^{2}$ test, a maximum likelihood-based Satorra-Bentler corrected test (SB), the asymptotically distribution free (ADF) test, and the two sample-size adjusted ADF methods due to Yuan and Bentler (YB1, YB2). The study used the open source software $R$ ( $R$ Core Team, 2014). The simulation 


\section{FURTHER EXPLORING ROBUST MEANS MODELING}

results were organized with the SimDesign package (Chalmers, 2016) and the RMM models were evaluated using lavaan (Rosseel, 2012).

Several variables were investigated in the simulation study, including the number of groups ( $K=2$ or 4 ), mean pattern (for investigating Type I error rates and power), sample sizes, population distribution shapes, and variance heterogeneity. Average group sample sizes were 10, 50 and 200 with both equal and unequal sample size conditions. Both equal and unequal variance conditions were included. The largest to smallest variance ratio was 16:1, which represents extreme levels of variance heterogeneity (Keselman et al., 1998). Two different heterogeneous variance conditions were included, one for a positively paired variance and sample size and one with a negatively paired variance and sample size. In addition to generating data from the normal (Gaussian) distribution, we simulated data from the $\chi^{2}$ distribution (with $3 d f$, skewness $=1.64$, kurtosis $=4.00$ ) and the $g$ and $h$ distribution (Hoaglin, 1985) with a positively skewed distribution $(g=1, h=0$, skewness $=6.18$, kurtosis $=113.94)$ and its negatively skewed counterpart $(g=-1, h=0)$. These distributions are expected to represent the moderate $\left(\chi^{2}\right)$ to extremely skewed $(g / h)$ distributions that behavioural science researchers would encounter (Wilcox, 1995). We explored conditions where all groups have the same population distribution shape, as well as conditions with mixtures of population distribution shapes (e.g., first population normal and second population positively skewed for $K=2)$.

In total, 420 unique conditions (300 conditions with the $g$ and $h$ distribution and 120 conditions with the $\chi^{2}$ distribution) were explored. The conditions for the simulation study are presented in Table 1 and were selected to match common design conditions in psychological research. To generate pseudo-random normal variates, we used the R function 'rnorm' (R Development Core Team, 2016). If $Z_{\mathrm{ij}}$ is a standard normal variate, then $X_{\mathrm{ij}}=\mu_{\mathrm{j}}+\sigma_{\mathrm{j}} \mathrm{Z}_{\mathrm{ij}}$ is a normal variate with mean equal to $\mu_{\mathrm{j}}$ and standard deviation equal to $\sigma_{\mathrm{j}}$. To generate data from a $g$ - and $h$-distribution, standard unit normal variables $\left(Z_{\mathrm{ij}}\right)$ were converted to the random variable:

$$
X_{i j}=\frac{e^{g Z_{i j}}-1}{g} e^{\frac{h Z_{i j}^{2}}{2}},
$$

where $g=1 /-1$ and $h=0$. To obtain a distribution with standard deviation $\sigma_{\mathrm{j}}$, each $\mathrm{X}_{\mathrm{ij}}$ was multiplied by a value of $\sigma_{j}$ (from Table 1$)$. It is important to note that this does not affect the value of the null hypothesis when $g=0$ (see Wilcox, 1994). However, when $g>0$, the population mean for a $g$ - and $h$-variable is:

$$
\mu_{g h}=\frac{1}{\sqrt{g(1-h)}}\left(e^{\frac{g^{2}}{2(h-1)}}-1\right) .
$$




\section{ALYSSA COUNSELL ET AL.}

Thus, for those conditions where $g>0, \mu_{\mathrm{gh}}$ was first subtracted from $\mathrm{X}_{\mathrm{ij}}$ before multiplying by $\sigma_{j}$. When working with trimmed means, the proportion of observations trimmed from each tail of the distribution was set at .2, and the population trimmed mean for the $j$ th group was also subtracted from the variate before multiplying by $\sigma_{\mathrm{j}}$. Lastly, it should be noted that the standard deviation of a $g$ - and $h$-distribution is not equal to one, and thus the values enumerated in Table 1 reflect only the amount that each random variable is multiplied by and not the actual values of the standard deviations (see Wilcox, 1994).

The nominal Type I error rate $(\alpha)$ was set at .05 for all conditions. There were 5000 replications conducted for each condition.

\section{Results}

Due to the large number of conditions, only a subset of the results is presented below. Specifically, we present the results for the moderate (average $n=50$ ) and the large (average $n=200$ ) sample size condition with four groups for each of the distribution types. These conditions were chosen to highlight any simulation conditions that had an effect on the Type I error rates. The results when $K=2$ mirror those when $K=4$. The full simulation results can be obtained from the first author.

Table 1. Simulation Conditions

\begin{tabular}{lcc}
\hline $\begin{array}{l}\text { Distributions } \\
\text { Dist. Patterns } \\
\text { T1 Mean Pattern }\end{array}$ & $\begin{array}{l}\text { Normal, } g \text { and } h \text { distribution (Positive, Negative Skew), } \chi^{2} \\
\text { Same Distribution Shape or Different for Half of the Groups } \\
\text { All population means }=0\end{array}$ & \multicolumn{1}{c}{$K=4$} \\
\hline Variance Pattern & 1,1 or 1,16 & $1,1,1,1$ or $1,4,9,16$ \\
Avg. $n=10$ & 10,$10 ; 4,16 ; 16,4$ & $10,10,10,10 ; 4,8,12,16 ;$ \\
Power Mean Pattern & $0,1.325$ & $16,12,8,4$ \\
Avg $n=50$ & 50,$50 ; 20,80 ; 80,20$ & $0,0.493,0.986,1.479$ \\
Power Mean Pattern & $0, .566$ & $50,50,50,50 ; 20,40,60,80 ;$ \\
Avg. $n=200$ & 200,$200 ; 80,320 ; 320,80$ & $0,0.211,0.422,0.633$ \\
& & $200,200,200,200 ;$ \\
Power Mean Pattern & $0, .281$ & $80,160,240,320 ;$ \\
\end{tabular}

Note: The mean patterns for power conditions were calculated for each of the three sample size conditions such that the power would be approximately .80 under normality, equal $n s$ and equal group variance. For Type I error rates, the raw population means were zero, but the trimmed population means were used for calculating Type I error rates for the trimmed Welch test. 


\section{FURTHER EXPLORING ROBUST MEANS MODELING}

\section{Estimation Issues for the RMM Methods}

Nonconvergence rates were minimal for RMM models as they converged in over $99.9 \%$ of the replications across all of the conditions. However, with smaller sample sizes (average $n$ of 10 ) the ADF methods exhibited problems with nonpositive definite matrices in the majority of conditions (rates as high as $88 \%$ ). This was no longer an issue when the average $n$ per group increased to 50 or 200 .

\section{Type I Error Rates}

The nominal Type I error rate was set at .05 for all investigated conditions and empirical rates were considered acceptable if they fell within Bradley's (1978) liberal bounds (i.e., $\alpha+/-.5 \alpha$ ). All of the tests were found to have accurate Type I error rates when all of the groups' data follow a normal distribution with equal group variance and sample sizes. However, once the groups' data did not follow a normal distribution (e.g., extremely positively or negatively skewed), many of the investigated tests no longer demonstrated accurate error rates. The only method found to maintain accurate empirical Type I error rates across all of the investigated conditions was the trimmed Welch ANOVA.

$\boldsymbol{g}$ and $\boldsymbol{h}$ distribution. Displayed in Tables 2 and 3 are the empirical Type I error rates for each of the tests when data were generated from the $g$ and $h$ distributions for average group sample sizes of 50 and 200, respectively. The accuracy of the Type I error rates for the RMM methods improves as sample size increases. When the average sample size per group was 50 (Table 2), the RMM methods' empirical error rates were found to be more liberal than the nominal $\alpha$ level under several simulation conditions. For example, in cases where the sample size and variance were negatively paired, rates were as high as .180 when the groups' distribution shapes were the same, and as high as .183 when the distribution shapes differed. When the average group sample size increased to 200 (see Table 3), the Type I error rates for the RMM methods become closer to the nominal $\alpha$ level, but are still somewhat liberal (e.g., as high as .10 in negative pairing conditions). As demonstrated in the tables, the error rates for the RMM approaches were very similar to one another regardless of the method used (e.g., traditional ML versus YB2).

$\chi^{2}$ distribution. Displayed in Tables 4 and 5 are the empirical error rates when data follow a $\chi^{2}$ distribution with three degrees of freedom for average group sample sizes of 50 and 200, respectively. The most notable finding is that the Type I error rates 


\section{ALYSSA COUNSELL ET AL.}

Table 2. $g$ and $h$ Distribution: Omnibus Type I Error Rates for $K=4$ and Average $n=50$

\begin{tabular}{|c|c|c|c|c|c|c|c|c|c|c|}
\hline Distribution & $n$ & $\sigma$ & ANOVA & Welch & T Welch & $M L$ & SB & ADF & YB1 & YB2 \\
\hline \multirow[t]{5}{*}{$0,0,0,0$} & $50,50,50,50$ & $1,1,1,1$ & 0.055 & 0.053 & 0.056 & 0.059 & 0.059 & 0.063 & 0.054 & 0.057 \\
\hline & $50,50,50,50$ & $1,2,3,4$ & 0.067 & 0.051 & 0.058 & 0.055 & 0.055 & 0.057 & 0.052 & 0.054 \\
\hline & $20,40,60,80$ & $1,1,1,1$ & 0.048 & 0.051 & 0.057 & 0.058 & 0.058 & 0.063 & 0.055 & 0.056 \\
\hline & $20,40,60,80$ & $1,2,3,4$ & 0.017 & 0.049 & 0.052 & 0.052 & 0.052 & 0.055 & 0.049 & 0.051 \\
\hline & $80,60,40,20$ & $1,2,3,4$ & 0.202 & 0.052 & 0.062 & 0.058 & 0.058 & 0.064 & 0.057 & 0.058 \\
\hline \multirow[t]{5}{*}{$1,1,1,1$} & $50,50,50,50$ & $1,1,1,1$ & 0.041 & 0.059 & 0.049 & 0.067 & 0.067 & 0.068 & 0.060 & 0.063 \\
\hline & $50,50,50,50$ & $1,2,3,4$ & 0.075 & 0.102 & 0.062 & 0.107 & 0.107 & 0.112 & 0.103 & 0.105 \\
\hline & $20,40,60,80$ & $1,1,1,1$ & 0.049 & 0.075 & 0.059 & 0.087 & 0.087 & 0.090 & 0.081 & 0.084 \\
\hline & $20,40,60,80$ & $1,2,3,4$ & 0.026 & 0.071 & 0.048 & 0.078 & 0.078 & 0.082 & 0.073 & 0.076 \\
\hline & $80,60,40,20$ & $1,2,3,4$ & 0.197 & 0.156 & 0.075 & 0.169 & 0.169 & 0.180 & 0.166 & 0.172 \\
\hline \multirow[t]{5}{*}{$2,2,2,2$} & $50,50,50,50$ & $1,1,1,1$ & 0.043 & 0.057 & 0.050 & 0.066 & 0.066 & 0.069 & 0.058 & 0.061 \\
\hline & $50,50,50,50$ & $1,2,3,4$ & 0.069 & 0.101 & 0.061 & 0.108 & 0.108 & 0.112 & 0.102 & 0.105 \\
\hline & $20,40,60,80$ & $1,1,1,1$ & 0.048 & 0.081 & 0.061 & 0.093 & 0.093 & 0.098 & 0.089 & 0.091 \\
\hline & $20,40,60,80$ & $1,2,3,4$ & 0.025 & 0.067 & 0.053 & 0.074 & 0.074 & 0.076 & 0.069 & 0.072 \\
\hline & $80,60,40,20$ & $1,2,3,4$ & 0.202 & 0.150 & 0.075 & 0.163 & 0.163 & 0.171 & 0.164 & 0.167 \\
\hline \multirow[t]{5}{*}{$0,0,1,1$} & $50,50,50,50$ & $1,1,1,1$ & 0.048 & 0.065 & 0.046 & 0.071 & 0.071 & 0.073 & 0.066 & 0.068 \\
\hline & $50,50,50,50$ & $1,2,3,4$ & 0.077 & 0.110 & 0.060 & 0.117 & 0.117 & 0.121 & 0.112 & 0.115 \\
\hline & $20,40,60,80$ & $1,1,1,1$ & 0.056 & 0.050 & 0.048 & 0.055 & 0.055 & 0.061 & 0.054 & 0.056 \\
\hline & $20,40,60,80$ & $1,2,3,4$ & 0.029 & 0.082 & 0.054 & 0.091 & 0.091 & 0.094 & 0.085 & 0.087 \\
\hline & $80,60,40,20$ & $1,2,3,4$ & 0.184 & 0.143 & 0.068 & 0.151 & 0.151 & 0.160 & 0.151 & 0.152 \\
\hline \multirow[t]{5}{*}{$0,0,2,2$} & $50,50,50,50$ & $1,1,1,1$ & 0.050 & 0.063 & 0.049 & 0.070 & 0.070 & 0.072 & 0.065 & 0.067 \\
\hline & $50,50,50,50$ & $1,2,3,4$ & 0.080 & 0.112 & 0.059 & 0.120 & 0.120 & 0.124 & 0.113 & 0.117 \\
\hline & $20,40,60,80$ & $1,1,1,1$ & 0.064 & 0.059 & 0.059 & 0.068 & 0.068 & 0.074 & 0.066 & 0.069 \\
\hline & $20,40,60,80$ & $1,2,3,4$ & 0.026 & 0.072 & 0.051 & 0.078 & 0.078 & 0.080 & 0.075 & 0.076 \\
\hline & $80,60,40,20$ & $1,2,3,4$ & 0.195 & 0.156 & 0.069 & 0.165 & 0.165 & 0.175 & 0.165 & 0.167 \\
\hline \multirow[t]{5}{*}{$1,1,2,2$} & $50,50,50,50$ & $1,1,1,1$ & 0.060 & 0.126 & 0.054 & 0.133 & 0.133 & 0.139 & 0.128 & 0.131 \\
\hline & $50,50,50,50$ & $1,2,3,4$ & 0.090 & 0.144 & 0.066 & 0.151 & 0.151 & 0.158 & 0.146 & 0.149 \\
\hline & $20,40,60,80$ & $1,1,1,1$ & 0.064 & 0.148 & 0.062 & 0.157 & 0.157 & 0.162 & 0.154 & 0.156 \\
\hline & $20,40,60,80$ & $1,2,3,4$ & 0.029 & 0.131 & 0.060 & 0.142 & 0.142 & 0.145 & 0.134 & 0.138 \\
\hline & $80,60,40,20$ & $1,2,3,4$ & 0.200 & 0.163 & 0.075 & 0.174 & 0.174 & 0.183 & 0.175 & 0.177 \\
\hline
\end{tabular}

Note: Dist $=0$ is the normal distribution, Dist $=1$ is a positively skewed distribution with $g=1$ and $h=0$, and Dist $=2$ is the negatively skewed distribution for $g=1$ and $h=0$. Rates outside of Bradley's liberal bounds are bolded.

for all of the procedures are better than those observed in similar conditions but with data generated from the $g$ and $h$ distribution. The RMM methods' error rates improve with the larger sample size condition whereby they only fall outside of Bradley's liberal bounds when the average $n$ is 50 in the negative pairing conditions, and are still less than $2 \alpha$. When the sample size increases to 200 , the RMM error rates are 


\section{FURTHER EXPLORING ROBUST MEANS MODELING}

Table 3. $g$ and $h$ Distribution: Omnibus Type I Error Rates for $K=4$ and average $n=200$

\begin{tabular}{|c|c|c|c|c|c|c|c|c|c|c|}
\hline Distrib & $\mathrm{n}$ & $\sigma$ & ANOVA & Welch & T Welch & $M L$ & SB & ADF & YB1 & YB2 \\
\hline \multirow[t]{5}{*}{$0,0,0,0$} & $200,200,200,200$ & $1,1,1,1$ & 0.050 & 0.052 & 0.054 & 0.052 & 0.052 & 0.052 & 0.052 & 0.052 \\
\hline & $200,200,200,200$ & $1,2,3,4$ & 0.073 & 0.050 & 0.051 & 0.052 & 0.052 & 0.053 & 0.051 & 0.051 \\
\hline & $80,160,240,320$ & $1,1,1,1$ & 0.057 & 0.056 & 0.052 & 0.057 & 0.057 & 0.058 & 0.057 & 0.058 \\
\hline & $80,160,240,320$ & $1,2,3,4$ & 0.019 & 0.050 & 0.050 & 0.051 & 0.051 & 0.052 & 0.050 & 0.050 \\
\hline & $320,240,160,80$ & $1,2,3,4$ & 0.195 & 0.051 & 0.051 & 0.052 & 0.052 & 0.053 & 0.052 & 0.052 \\
\hline \multirow[t]{5}{*}{$1,1,1,1$} & $200,200,200,200$ & $1,1,1,1$ & 0.048 & 0.058 & 0.048 & 0.060 & 0.060 & 0.061 & 0.058 & 0.059 \\
\hline & $200,200,200,200$ & $1,2,3,4$ & 0.068 & 0.073 & 0.050 & 0.074 & 0.074 & 0.075 & 0.073 & 0.073 \\
\hline & $80,160,240,320$ & $1,1,1,1$ & 0.047 & 0.067 & 0.049 & 0.068 & 0.068 & 0.068 & 0.067 & 0.067 \\
\hline & $80,160,240,320$ & $1,2,3,4$ & 0.021 & 0.061 & 0.050 & 0.062 & 0.062 & 0.062 & 0.061 & 0.061 \\
\hline & $320,240,160,80$ & $1,2,3,4$ & 0.189 & 0.098 & 0.057 & 0.100 & 0.100 & 0.101 & 0.100 & 0.100 \\
\hline \multirow[t]{5}{*}{$2,2,2,2$} & $200,200,200,200$ & $1,1,1,1$ & 0.046 & 0.055 & 0.057 & 0.058 & 0.058 & 0.058 & 0.056 & 0.056 \\
\hline & $200,200,200,200$ & $1,2,3,4$ & 0.071 & 0.076 & 0.059 & 0.078 & 0.078 & 0.079 & 0.077 & 0.078 \\
\hline & $80,160,240,320$ & $1,1,1,1$ & 0.046 & 0.067 & 0.050 & 0.069 & 0.069 & 0.070 & 0.068 & 0.069 \\
\hline & $80,160,240,320$ & $1,2,3,4$ & 0.024 & 0.062 & 0.049 & 0.064 & 0.064 & 0.065 & 0.063 & 0.063 \\
\hline & $320,240,160,80$ & $1,2,3,4$ & 0.201 & 0.095 & 0.056 & 0.098 & 0.098 & 0.100 & 0.097 & 0.098 \\
\hline \multirow[t]{5}{*}{$0,0,1,1$} & $200,200,200,200$ & $1,1,1,1$ & 0.052 & 0.063 & 0.050 & 0.065 & 0.065 & 0.066 & 0.063 & 0.063 \\
\hline & $200,200,200,200$ & $1,2,3,4$ & 0.070 & 0.074 & 0.050 & 0.075 & 0.075 & 0.075 & 0.074 & 0.075 \\
\hline & $80,160,240,320$ & $1,1,1,1$ & 0.057 & 0.055 & 0.053 & 0.058 & 0.058 & 0.059 & 0.057 & 0.057 \\
\hline & $80,160,240,320$ & $1,2,3,4$ & 0.024 & 0.068 & 0.052 & 0.070 & 0.070 & 0.071 & 0.068 & 0.069 \\
\hline & $320,240,160,80$ & $1,2,3,4$ & 0.194 & 0.095 & 0.051 & 0.097 & 0.097 & 0.100 & 0.097 & 0.098 \\
\hline \multirow[t]{5}{*}{$0,0,2,2$} & $200,200,200,200$ & $1,1,1,1$ & 0.049 & 0.056 & 0.055 & 0.057 & 0.057 & 0.057 & 0.056 & 0.056 \\
\hline & $200,200,200,200$ & $1,2,3,4$ & 0.063 & 0.052 & 0.050 & 0.054 & 0.054 & 0.056 & 0.053 & 0.054 \\
\hline & $80,160,240,320$ & $1,1,1,1$ & 0.052 & 0.078 & 0.056 & 0.080 & 0.080 & 0.082 & 0.079 & 0.079 \\
\hline & $80,160,240,320$ & $1,2,3,4$ & 0.024 & 0.055 & 0.051 & 0.057 & 0.057 & 0.057 & 0.055 & 0.056 \\
\hline & $320,240,160,80$ & $1,2,3,4$ & 0.197 & 0.058 & 0.050 & 0.061 & 0.061 & 0.062 & 0.060 & 0.061 \\
\hline \multirow[t]{5}{*}{$1,1,2,2$} & $200,200,200,200$ & $1,1,1,1$ & 0.048 & 0.076 & 0.052 & 0.077 & 0.077 & 0.078 & 0.076 & 0.076 \\
\hline & $200,200,200,200$ & $1,2,3,4$ & 0.074 & 0.085 & 0.053 & 0.086 & 0.086 & 0.087 & 0.085 & 0.086 \\
\hline & $80,160,240,320$ & $1,1,1,1$ & 0.054 & 0.096 & 0.053 & 0.097 & 0.097 & 0.099 & 0.096 & 0.097 \\
\hline & $80,160,240,320$ & $1,2,3,4$ & 0.023 & 0.086 & 0.054 & 0.087 & 0.087 & 0.088 & 0.086 & 0.087 \\
\hline & $320,240,160,80$ & $1,2,3,4$ & 0.211 & 0.106 & 0.062 & 0.107 & 0.107 & 0.110 & 0.108 & 0.108 \\
\hline
\end{tabular}


ALYSSA COUNSELL ET AL.

Table 4. $\chi^{2}$ Distribution: Omnibus Type I Error Rates for $K=4$ and Average $n=50$

\begin{tabular}{ccccccccccc}
\hline Distribution & $n$ & $\sigma$ & ANOVA & Welch & T Welch & ML & SB & ADF & YB1 & YB2 \\
\hline $\mathbf{1 , 1 , 1 , 1}$ & $50,50,50,50$ & $1,1,1,1$ & 0.050 & 0.057 & 0.051 & 0.063 & 0.063 & 0.067 & 0.059 & 0.061 \\
& $50,50,50,50$ & $1,2,3,4$ & 0.067 & 0.065 & 0.054 & 0.068 & 0.068 & 0.071 & 0.065 & 0.067 \\
& $20,40,60,80$ & $1,1,1,1$ & 0.053 & 0.060 & 0.056 & 0.070 & 0.070 & 0.074 & 0.066 & 0.068 \\
& $20,40,60,80$ & $1,2,3,4$ & $\mathbf{0 . 0 2 3}$ & 0.054 & 0.056 & 0.058 & 0.058 & 0.060 & 0.055 & 0.057 \\
$0,0,1,1$ & $80,60,40,20$ & $1,2,3,4$ & $\mathbf{0 . 2 1 2}$ & $\mathbf{0 . 0 8 3}$ & 0.070 & $\mathbf{0 . 0 9 2}$ & $\mathbf{0 . 0 9 2}$ & $\mathbf{0 . 1 0 1}$ & $\mathbf{0 . 0 9 2}$ & $\mathbf{0 . 0 9 5}$ \\
& $50,50,50,50$ & $1,1,1,1$ & 0.051 & 0.054 & 0.049 & 0.059 & 0.059 & 0.062 & 0.055 & 0.057 \\
& $50,50,50,50$ & $1,2,3,4$ & 0.072 & 0.069 & 0.051 & 0.073 & 0.073 & $\mathbf{0 . 0 7 6}$ & 0.070 & 0.072 \\
& $20,40,60,80$ & $1,1,1,1$ & 0.046 & 0.049 & 0.054 & 0.058 & 0.058 & 0.064 & 0.056 & 0.059 \\
& $20,40,60,80$ & $1,2,3,4$ & $\mathbf{0 . 0 2 1}$ & 0.055 & 0.058 & 0.061 & 0.061 & 0.065 & 0.056 & 0.058 \\
& $80,60,40,20$ & $1,2,3,4$ & $\mathbf{0 . 2 0 5}$ & $\mathbf{0 . 0 7 7}$ & 0.064 & $\mathbf{0 . 0 8 3}$ & $\mathbf{0 . 0 8 3}$ & $\mathbf{0 . 0 9 3}$ & 0.085 & 0.088 \\
\hline
\end{tabular}

Note: Dist $=1$ is the $\chi^{2}$ distribution with 3 degrees of freedom, and Dist $=0$ is the normal distribution

accurate in all of the variance-sample size pairings. The trimmed Welch procedure's error rates are accurate across all conditions regardless of sample size.

\section{Power Rates}

Fan and Hancock (2012) did not report power results for the trimmed Welch because their simulation study reported inaccurate error rates for the test. This was not the case in this simulation, and therefore power results are presented for the same conditions investigated above. Power rates are in bold when the error rates for the same conditions in the previous section fell outside of Bradley's liberal bounds. The population means are different for the average $n=50$ and average $n=200$ conditions because the mean pattern reflects power rates of approximately .80 for homoscedastic and normally distributed data with equal sample sizes per group. Taking this approach means that there is no power increase by increasing sample size from 50 to 200, because the conditions use different population means to assess power.

$g$ and $\boldsymbol{h}$ distribution. Presented in Tables 6 and 7 are the power results under the same conditions used for Type I error rates for the two sample sizes when data follow the $g$ and $h$ distribution. When all assumptions have been met, the trimmed Welch has somewhat lower power than the other procedures by about $8 \%$ (which is expected because of the reduced effective sample size with trimming). With skewed data, however, the trimmed Welch demonstrates comparable, and for many conditions, superior power rates compared to the RMM methods. This pattern of results 
FURTHER EXPLORING ROBUST MEANS MODELING

Table 5. $\chi^{2}$ Distribution: Omnibus Type I Error Rates for $K=4$ and Average $n=200$

\begin{tabular}{ccccccccccc}
\hline Distribution & $n$ & $\sigma$ & ANOVA & Welch & T Welch & ML & SB & ADF & YB1 & YB2 \\
\hline \multirow{1}{1,1,1,1}{} & $200,200,200,200$ & $1,1,1,1$ & 0.051 & 0.053 & 0.052 & 0.054 & 0.054 & 0.054 & 0.053 & 0.053 \\
& $200,200,200,200$ & $1,2,3,4$ & 0.069 & 0.055 & 0.049 & 0.056 & 0.056 & 0.056 & 0.055 & 0.056 \\
& $80,160,240,320$ & $1,1,1,1$ & 0.052 & 0.055 & 0.050 & 0.057 & 0.057 & 0.058 & 0.057 & 0.057 \\
& $80,160,240,320$ & $1,2,3,4$ & $\mathbf{0 . 0 2 1}$ & 0.049 & 0.053 & 0.049 & 0.049 & 0.051 & 0.049 & 0.050 \\
$0,0,1,1$ & $320,240,160,80$ & $1,2,3,4$ & $\mathbf{0 . 1 9 6}$ & 0.058 & 0.050 & 0.059 & 0.059 & 0.060 & 0.060 & 0.060 \\
& $200,200,200,200$ & $1,1,1,1$ & 0.048 & 0.049 & 0.048 & 0.050 & 0.050 & 0.051 & 0.049 & 0.050 \\
& $200,200,200,200$ & $1,2,3,4$ & 0.065 & 0.048 & 0.051 & 0.049 & 0.049 & 0.049 & 0.048 & 0.048 \\
& $80,160,240,320$ & $1,1,1,1$ & 0.051 & 0.051 & 0.057 & 0.053 & 0.053 & 0.055 & 0.053 & 0.053 \\
& $80,160,240,320$ & $1,2,3,4$ & $\mathbf{0 . 0 1 9}$ & 0.053 & 0.046 & 0.055 & 0.055 & 0.055 & 0.054 & 0.054 \\
& $320,240,160,80$ & $1,2,3,4$ & $\mathbf{0 . 2 0 1}$ & 0.061 & 0.055 & 0.064 & 0.064 & 0.065 & 0.064 & 0.064 \\
\hline
\end{tabular}

was also true when the RMM methods were found to have liberal error rates. For all procedures, unequal variances drastically decreases power to detect population mean differences. As with Type I error rates, the RMM procedures exhibited similar power rates to one another, such that one procedure did not consistently outperform the others.

$\chi^{2}$ distribution. Presented in Tables 8 and 9 are the power results for outcomes that follow a $\chi^{2}$ distribution with three $d f$. A similar pattern of power results was observed as those discussed above when data were generated from the $g$ and $h$ distribution. The power results for the trimmed Welch and RMM approaches were similar across the conditions and the different RMM procedures were almost identical.

\section{Conclusion}

Given the popularity of comparing mean differences and prevalence of assumption violation in research in the behavioural sciences (e.g., Blanca et al., 2011; Golinski \& Cribbie, 2009; Keselman et al., 1998; Micceri, 1989), it is important that researchers have viable alternatives to the traditional ANOVA. A recent study proposed another robust statistical tool for comparing mean differences called robust means modeling (Fan \& Hancock, 2012). Given their somewhat surprising results regarding the trimmed Welch test, the aim of the current study was to replicate their findings and extend this area of research in a few important ways; namely by examining Type I error and power rates with other families of distributions (e.g., $\left.g / h, \chi^{2}\right)$ and exploring the performance when the populations have differing distribution shapes. 


\section{ALYSSA COUNSELL ET AL.}

Table 6. Power Results for $g$ and $h$ distributions with $K=4$ and average $n=50$

\begin{tabular}{|c|c|c|c|c|c|c|c|c|c|c|}
\hline Distribution & $n$ & $\sigma$ & ANOVA & Welch & T Welch & ML & SB & ADF & YB1 & YB2 \\
\hline \multirow[t]{5}{*}{$0,0,0,0$} & $50,50,50,50$ & $1,1,1,1$ & 0.810 & 0.803 & 0.731 & 0.814 & 0.814 & 0.818 & 0.805 & 0.809 \\
\hline & $50,50,50,50$ & $1,2,3,4$ & 0.156 & 0.190 & 0.176 & 0.206 & 0.206 & 0.214 & 0.194 & 0.200 \\
\hline & $20,40,60,80$ & $1,1,1,1$ & 0.687 & 0.675 & 0.595 & 0.696 & 0.696 & 0.708 & 0.690 & 0.697 \\
\hline & $20,40,60,80$ & $1,2,3,4$ & 0.054 & 0.187 & 0.165 & 0.203 & 0.203 & 0.209 & 0.191 & 0.195 \\
\hline & $80,60,40,20$ & $1,2,3,4$ & 0.311 & 0.150 & 0.138 & 0.165 & 0.165 & 0.177 & 0.164 & 0.169 \\
\hline \multirow[t]{5}{*}{$1,1,1,1$} & $50,50,50,50$ & $1,1,1,1$ & 0.833 & 0.881 & 0.999 & 0.888 & 0.888 & 0.893 & 0.882 & 0.885 \\
\hline & $50,50,50,50$ & $1,2,3,4$ & 0.111 & 0.116 & 0.488 & 0.131 & 0.131 & 0.138 & 0.122 & 0.126 \\
\hline & $20,40,60,80$ & $1,1,1,1$ & 0.756 & 0.846 & 0.984 & 0.858 & 0.858 & 0.862 & 0.853 & 0.856 \\
\hline & $20,40,60,80$ & $1,2,3,4$ & 0.029 & 0.217 & 0.513 & 0.232 & 0.232 & 0.237 & 0.219 & 0.225 \\
\hline & $80,60,40,20$ & $1,2,3,4$ & 0.275 & 0.085 & 0.320 & 0.101 & 0.101 & 0.109 & 0.099 & 0.102 \\
\hline \multirow[t]{5}{*}{$2,2,2,2$} & $50,50,50,50$ & $1,1,1,1$ & 0.820 & 0.875 & 0.999 & 0.882 & 0.882 & 0.884 & 0.876 & 0.879 \\
\hline & $50,50,50,50$ & $1,2,3,4$ & 0.252 & 0.445 & 0.598 & 0.457 & 0.457 & 0.465 & 0.448 & 0.454 \\
\hline & $20,40,60,80$ & $1,1,1,1$ & 0.726 & 0.779 & 0.997 & 0.795 & 0.795 & 0.802 & 0.790 & 0.792 \\
\hline & $20,40,60,80$ & $1,2,3,4$ & 0.119 & 0.362 & 0.576 & 0.374 & 0.374 & 0.383 & 0.367 & 0.373 \\
\hline & $80,60,40,20$ & $1,2,3,4$ & 0.407 & 0.455 & 0.506 & 0.469 & 0.469 & 0.481 & 0.467 & 0.471 \\
\hline \multirow[t]{5}{*}{$0,0,1,1$} & $50,50,50,50$ & $1,1,1,1$ & 0.846 & 0.841 & 0.951 & 0.855 & 0.855 & 0.860 & 0.844 & 0.849 \\
\hline & $50,50,50,50$ & $1,2,3,4$ & 0.100 & 0.112 & 0.326 & 0.124 & 0.124 & 0.129 & 0.115 & 0.118 \\
\hline & $20,40,60,80$ & $1,1,1,1$ & 0.740 & 0.717 & 0.857 & 0.744 & 0.744 & 0.757 & 0.733 & 0.742 \\
\hline & $20,40,60,80$ & $1,2,3,4$ & 0.030 & 0.127 & 0.289 & 0.138 & 0.138 & 0.145 & 0.131 & 0.134 \\
\hline & $80,60,40,20$ & $1,2,3,4$ & 0.250 & 0.100 & 0.231 & 0.120 & 0.120 & 0.126 & 0.114 & 0.117 \\
\hline \multirow[t]{5}{*}{$0,0,2,2$} & $50,50,50,50$ & $1,1,1,1$ & 0.796 & 0.845 & 0.911 & 0.855 & 0.855 & 0.857 & 0.848 & 0.852 \\
\hline & $50,50,50,50$ & $1,2,3,4$ & 0.252 & 0.414 & 0.440 & 0.426 & 0.426 & 0.433 & 0.416 & 0.422 \\
\hline & $20,40,60,80$ & $1,1,1,1$ & 0.711 & 0.722 & 0.821 & 0.739 & 0.739 & 0.749 & 0.734 & 0.739 \\
\hline & $20,40,60,80$ & $1,2,3,4$ & 0.118 & 0.347 & 0.335 & 0.360 & 0.360 & 0.366 & 0.350 & 0.354 \\
\hline & $80,60,40,20$ & $1,2,3,4$ & 0.396 & 0.414 & 0.376 & 0.434 & 0.434 & 0.447 & 0.433 & 0.438 \\
\hline \multirow[t]{5}{*}{$1,1,2,2$} & $50,50,50,50$ & $1,1,1,1$ & 0.768 & 0.868 & 0.989 & 0.874 & 0.874 & 0.876 & 0.868 & 0.871 \\
\hline & $50,50,50,50$ & $1,2,3,4$ & 0.263 & 0.431 & 0.547 & 0.439 & 0.439 & 0.448 & 0.434 & 0.437 \\
\hline & $20,40,60,80$ & $1,1,1,1$ & 0.704 & 0.826 & 0.968 & 0.835 & 0.835 & 0.841 & 0.832 & 0.835 \\
\hline & $20,40,60,80$ & $1,2,3,4$ & 0.120 & 0.438 & 0.546 & 0.451 & 0.451 & 0.457 & 0.441 & 0.445 \\
\hline & $80,60,40,20$ & $1,2,3,4$ & 0.412 & 0.416 & 0.475 & 0.432 & 0.432 & 0.444 & 0.430 & 0.435 \\
\hline
\end{tabular}

Bolded values indicate conditions where the Type I error rates were unacceptable. 


\section{FURTHER EXPLORING ROBUST MEANS MODELING}

Table 7. Power Results for $g$ and $h$ distributions with $K=4$ and average $n=200$

\begin{tabular}{|c|c|c|c|c|c|c|c|c|c|c|}
\hline Distribution & $n$ & $\sigma$ & ANOVA & Welch & T Welch & $\mathrm{ML}$ & SB & ADF & YB1 & YB2 \\
\hline \multirow[t]{5}{*}{$0,0,0,0$} & $200,200,200,200$ & $1,1,1,1$ & 0.803 & 0.799 & 0.729 & 0.801 & 0.801 & 0.803 & 0.799 & 0.800 \\
\hline & $200,200,200,200$ & $1,2,3,4$ & 0.145 & 0.196 & 0.174 & 0.200 & 0.200 & 0.202 & 0.197 & 0.199 \\
\hline & $80,160,240,320$ & $1,1,1,1$ & 0.700 & 0.696 & 0.623 & 0.701 & 0.701 & 0.703 & 0.699 & 0.700 \\
\hline & $80,160,240,320$ & $1,2,3,4$ & 0.052 & 0.192 & 0.164 & 0.195 & 0.195 & 0.196 & 0.193 & 0.193 \\
\hline & $320,240,160,80$ & $1,2,3,4$ & 0.318 & 0.172 & 0.151 & 0.177 & 0.177 & 0.180 & 0.176 & 0.177 \\
\hline \multirow[t]{5}{*}{$1,1,1,1$} & $200,200,200,200$ & $1,1,1,1$ & 0.808 & 0.839 & 0.999 & 0.840 & 0.840 & 0.842 & 0.839 & 0.840 \\
\hline & $200,200,200,200$ & $1,2,3,4$ & 0.123 & 0.135 & 0.514 & 0.137 & 0.137 & 0.139 & 0.136 & 0.137 \\
\hline & $80,160,240,320$ & $1,1,1,1$ & 0.736 & 0.790 & 0.993 & 0.795 & 0.795 & 0.797 & 0.793 & 0.794 \\
\hline & $80,160,240,320$ & $1,2,3,4$ & 0.040 & 0.198 & 0.536 & 0.202 & 0.202 & 0.203 & 0.198 & 0.200 \\
\hline & $320,240,160,80$ & $1,2,3,4$ & 0.273 & 0.090 & 0.393 & 0.094 & 0.094 & 0.097 & 0.092 & 0.094 \\
\hline \multirow[t]{5}{*}{$2,2,2,2$} & $200,200,200,200$ & $1,1,1,1$ & 0.819 & 0.847 & 0.999 & 0.849 & 0.849 & 0.850 & 0.847 & 0.848 \\
\hline & $200,200,200,200$ & $1,2,3,4$ & 0.224 & 0.354 & 0.593 & 0.357 & 0.357 & 0.358 & 0.355 & 0.356 \\
\hline & $80,160,240,320$ & $1,1,1,1$ & 0.700 & 0.718 & 0.998 & 0.724 & 0.724 & 0.727 & 0.721 & 0.722 \\
\hline & $80,160,240,320$ & $1,2,3,4$ & 0.084 & 0.272 & 0.552 & 0.276 & 0.276 & 0.278 & 0.273 & 0.275 \\
\hline & $320,240,160,80$ & $1,2,3,4$ & 0.378 & 0.342 & 0.502 & 0.347 & 0.347 & 0.349 & 0.346 & 0.347 \\
\hline \multirow[t]{5}{*}{$0,0,1,1$} & $200,200,200,200$ & $1,1,1,1$ & 0.827 & 0.823 & 0.949 & 0.827 & 0.827 & 0.829 & 0.824 & 0.825 \\
\hline & $200,200,200,200$ & $1,2,3,4$ & 0.117 & 0.141 & 0.362 & 0.145 & 0.145 & 0.146 & 0.141 & 0.143 \\
\hline & $80,160,240,320$ & $1,1,1,1$ & 0.721 & 0.709 & 0.861 & 0.715 & 0.715 & 0.719 & 0.713 & 0.715 \\
\hline & $80,160,240,320$ & $1,2,3,4$ & 0.033 & 0.140 & 0.307 & 0.144 & 0.144 & 0.145 & 0.140 & 0.141 \\
\hline & $320,240,160,80$ & $1,2,3,4$ & 0.268 & 0.102 & 0.257 & 0.105 & 0.105 & 0.108 & 0.105 & 0.105 \\
\hline \multirow[t]{5}{*}{$0,0,2,2$} & $200,200,200,200$ & $1,1,1,1$ & 0.784 & 0.812 & 0.931 & 0.816 & 0.816 & 0.817 & 0.813 & 0.814 \\
\hline & $200,200,200,200$ & $1,2,3,4$ & 0.212 & 0.329 & 0.419 & 0.332 & 0.332 & 0.333 & 0.330 & 0.331 \\
\hline & $80,160,240,320$ & $1,1,1,1$ & 0.690 & 0.709 & 0.843 & 0.715 & 0.715 & 0.718 & 0.714 & 0.715 \\
\hline & $80,160,240,320$ & $1,2,3,4$ & 0.086 & 0.276 & 0.321 & 0.279 & 0.279 & 0.280 & 0.277 & 0.278 \\
\hline & $320,240,160,80$ & $1,2,3,4$ & 0.365 & 0.314 & 0.349 & 0.318 & 0.318 & 0.322 & 0.318 & 0.320 \\
\hline \multirow[t]{5}{*}{$1,1,2,2$} & $200,200,200,200$ & $1,1,1,1$ & 0.774 & 0.832 & 0.998 & 0.835 & 0.835 & 0.836 & 0.832 & 0.833 \\
\hline & $200,200,200,200$ & $1,2,3,4$ & 0.216 & 0.338 & 0.557 & 0.341 & 0.341 & 0.344 & 0.340 & 0.341 \\
\hline & $80,160,240,320$ & $1,1,1,1$ & 0.695 & 0.783 & 0.989 & 0.786 & 0.786 & 0.787 & 0.785 & 0.786 \\
\hline & $80,160,240,320$ & $1,2,3,4$ & 0.086 & 0.334 & 0.541 & 0.339 & 0.339 & 0.340 & 0.335 & 0.336 \\
\hline & $320,240,160,80$ & $1,2,3,4$ & 0.370 & 0.314 & 0.462 & 0.318 & 0.318 & 0.322 & 0.318 & 0.320 \\
\hline
\end{tabular}

Bolded values indicate conditions where the Type I error rates were unacceptable.

\section{RMM Procedures}

Although Fan and Hancock (2012) recommended the YB1 or YB2 approaches as the better performing tests, we found little deviation in the performance of the different RMM methods. Type I error rates for the procedures were similar and there was no noticeable power advantage of any other approaches under the conditions investigated. The degree of similarity between the regular ML approach and the 
ALYSSA COUNSELL ET AL.

Table 8. Power Rates for the $\chi^{2}$ distribution with $K=4$ and average $n=50$

\begin{tabular}{ccccccccccc}
\hline Distribution & $n$ & $\sigma$ & ANOVA & Welch & T Welch & ML & SB & ADF & YB1 & YB2 \\
\hline $\mathbf{1 , 1 , 1 , 1}$ & $50,50,50,50$ & $1,1,1,1$ & 0.802 & 0.802 & 0.827 & 0.814 & 0.814 & 0.820 & 0.803 & 0.809 \\
& $50,50,50,50$ & $1,2,3,4$ & 0.134 & 0.145 & 0.166 & 0.158 & 0.158 & 0.166 & 0.150 & 0.154 \\
& $20,40,60,80$ & $1,1,1,1$ & 0.715 & 0.733 & 0.714 & 0.751 & 0.751 & 0.758 & 0.743 & 0.747 \\
& $20,40,60,80$ & $1,2,3,4$ & $\mathbf{0 . 0 4 1}$ & 0.190 & 0.195 & 0.206 & 0.206 & 0.211 & 0.193 & 0.198 \\
$0,0,1,1$ & $80,60,40,20$ & $1,2,3,4$ & $\mathbf{0 . 2 8 8}$ & $\mathbf{0 . 0 9 3}$ & 0.122 & $\mathbf{0 . 1 0 7}$ & $\mathbf{0 . 1 0 7}$ & $\mathbf{0 . 1 1 6}$ & $\mathbf{0 . 1 0 4}$ & $\mathbf{0 . 1 0 9}$ \\
& $50,50,50,50$ & $1,1,1,1$ & 0.812 & 0.797 & 0.784 & 0.813 & 0.813 & 0.817 & 0.800 & 0.806 \\
& $50,50,50,50$ & $1,2,3,4$ & 0.119 & 0.140 & 0.167 & 0.154 & 0.154 & $\mathbf{0 . 1 6 1}$ & 0.144 & 0.149 \\
& $20,40,60,80$ & $1,1,1,1$ & 0.704 & 0.684 & 0.656 & 0.708 & 0.708 & 0.719 & 0.697 & 0.705 \\
& $20,40,60,80$ & $1,2,3,4$ & $\mathbf{0 . 0 3 9}$ & 0.154 & 0.169 & 0.169 & 0.169 & 0.175 & 0.159 & 0.164 \\
& $80,60,40,20$ & $1,2,3,4$ & $\mathbf{0 . 2 8 5}$ & $\mathbf{0 . 1 0 4}$ & 0.117 & $\mathbf{0 . 1 2 5}$ & $\mathbf{0 . 1 2 5}$ & $\mathbf{0 . 1 3 5}$ & $\mathbf{0 . 1 2 0}$ & $\mathbf{0 . 1 2 5}$ \\
\hline
\end{tabular}

Bolded values indicate conditions where the Type I error rates were unacceptable.

ADF methods was somewhat surprising because ML requires the assumption of conditionally normally distributed data. If one were to choose between the methods, the regular ML approach or Satorra-Bentler corrected ML test might therefore be preferable with smaller sample sizes because, according to our observations, they did not exhibit any problems with nonpositive definite matrices, whereas this was sometimes an issue for the ADF methods.

Distribution shape had an effect on the performance of the RMM methods. Empirical Type I error rates were better when data followed a $\chi^{2}$ (with three $d f$ ) distribution compared to the positively or negatively skewed $g$ and $h$ distribution. However, this may be due to severity of nonnormality as the $\chi^{2}$ distribution is less skewed than the

Table 9. Power Rates for the $\chi^{2}$ distribution with $K=4$ and average $n=200$

\begin{tabular}{ccccccccccc}
\hline Distribution & $n$ & $\sigma$ & ANOVA & Welch & TWelch & ML & SB & ADF & YB1 & YB2 \\
\hline $1,1,1,1$ & $200,200,200,200$ & $1,1,1,1$ & 0.806 & 0.803 & 0.841 & 0.806 & 0.806 & 0.807 & 0.804 & 0.804 \\
& $200,200,200,200$ & $1,2,3,4$ & 0.147 & 0.163 & 0.188 & 0.166 & 0.166 & 0.168 & 0.163 & 0.165 \\
& $80,160,240,320$ & $1,1,1,1$ & 0.692 & 0.700 & 0.743 & 0.706 & 0.706 & 0.708 & 0.703 & 0.705 \\
& $80,160,240,320$ & $1,2,3,4$ & $\mathbf{0 . 0 4 5}$ & 0.191 & 0.196 & 0.194 & 0.194 & 0.195 & 0.192 & 0.193 \\
& $320,240,160,80$ & $1,2,3,4$ & $\mathbf{0 . 3 1 5}$ & 0.130 & 0.143 & 0.134 & 0.134 & 0.136 & 0.133 & 0.135 \\
$0,0,1,1$ & $200,200,200,200$ & $1,1,1,1$ & 0.799 & 0.793 & 0.789 & 0.796 & 0.796 & 0.798 & 0.793 & 0.795 \\
& $200,200,200,200$ & $1,2,3,4$ & 0.136 & 0.160 & 0.176 & 0.165 & 0.165 & 0.167 & 0.161 & 0.163 \\
& $80,160,240,320$ & $1,1,1,1$ & 0.704 & 0.695 & 0.674 & 0.703 & 0.703 & 0.707 & 0.701 & 0.702 \\
& $80,160,240,320$ & $1,2,3,4$ & $\mathbf{0 . 0 4 0}$ & 0.166 & 0.185 & 0.170 & 0.170 & 0.172 & 0.166 & 0.168 \\
& $320,240,160,80$ & $1,2,3,4$ & $\mathbf{0 . 2 9 4}$ & 0.127 & 0.137 & 0.131 & 0.131 & 0.133 & 0.130 & 0.132 \\
\hline
\end{tabular}

Bolded values indicate conditions where the Type I error rates were unacceptable. 


\section{FURTHER EXPLORING ROBUST MEANS MODELING}

$g$ and $h$ distributions used in the current study. Sample size also influenced the empirical Type I error rates in that the tests became overly conservative with smaller sample sizes. Given that the methods use ML estimation and the ADF methods are notorious for requiring larger sample sizes, it is possible that the models produces more biased estimates in smaller sample sizes, which manifested in the poorer Type I error rates.

\section{Trimmed Welch versus RMM}

The most noteworthy finding is the difference between the performance of the trimmed Welch ANOVA and the RMM methods in the current study compared to what was reported in Fan and Hancock (2012). Specifically, they reported inconsistent and often extremely liberal Type I error rates for the trimmed Welch, whereas we found that the rates were very stable around the nominal $\alpha$ level. In fact, the trimmed Welch was the only procedure with empirical Type I error rates inside an acceptable range under all of the conditions tested. Our results regarding the Type I error rates of the Welch test on trimmed means agree with the results of several previous simulation studies including Cribbie, Wilcox, Bewell \& Keselman (2007), Cribbie et al. (2012), Lix and Keselman (2006), and Wilcox (1995), and therefore we are confident that the Welch test on trimmed means is not overly liberal with heteroscedastic and/or skewed distributions. Additionally, the trimmed Welch had comparable or higher power than the RMM tests, including conditions where the RMM's Type I error rates were more liberal than the nominal $\alpha$ rate.

One important consideration when comparing the trimmed Welch procedure to the RMM approaches is that of effect size. Reporting statistical significance tests does not allow for an indication of the magnitude of group differences. Published ANOVA results often include the raw group means (or mean differences) as the effect size measure when data are normally distributed (or have similar distribution shapes) and group variances are approximately equal. However, reporting raw mean differences may not be the best choice under assumption violations, as means are sensitive to nonnormality and outliers. Reporting trimmed means, however, is an intuitive and appropriate effect size when conducting the trimmed Welch. It has an easy interpretation for applied researchers who are accustomed to reporting means or mean differences. In contrast, an appropriate measure of effect size in conjunction with the RMM procedures is unclear. Reporting raw means with RMM methods is somewhat inconsistent as they do not account for the nonnormality or heterogeneity of the data. 


\section{ALYSSA COUNSELL ET AL.}

\section{References}

Alexander, R. A., \& Govern, D. M. (1994). A new and simpler approximation for ANOVA under variance heterogeneity. Journal of Educational Statistics, 19(2), 91-101. doi: 10.3102/10769986019002091

Algina, J., Oshima, T. C., \& Lin, W. Y. (1994). Type I error rates for Welch's test and James's second-order test under nonnormality and inequality of variance when there are two groups. Journal of Educational and Behavioral Statistics, 19(3), 275-291. doi: 10.3102/10769986019003275

Blanca, M. J., Arnau, J., Lopez-Montiel, D., Bono, R., \& Bendayan, R. (2011) Skewness and kurtosis in real data samples. Methodology, 9(2), 78-84. doi: 10.1027/1614-2241/a000057

Bollen, K. A. (1989). Structural Equations with Latent Variables. New York: Wiley. doi: 10.1002/9781118619179

Boneau, C. A. (1960). The effects of violations of assumptions underlying the t test. Psychological Bulletin, 57(1), 49-64. doi: 10.1037/h0041412

Box, G. E. P. (1954). Some theorems on quadratic forms applied in the study of analysis of variance problems, I. Effect of inequality of variance in the one-way classification. Annals of Mathematical Statistics, 25(2), 290-302. doi: 10.1214/aoms/1177728786

Bradley, J. V. (1978). Robustness? British Journal of Mathematical and Statistical Psychology, 31(2), 144-152. doi: 10.1111/j.2044-8317.1978.tb00581.x

Brown, M. B., \& Forsythe, A. B. (1974a). The ANOVA and multiple comparisons for data with heterogeneous variances. Biometrics, 30(4), 719-724. doi: 10.2307/2529238

Brown, M. B., \& Forsythe, A. B. (1974b). The small sample behavior of some statistics which test the equality of several means. Technometrics, 16(1), 129-132. doi: 10 $.1080 / 00401706.1974 .10489158$

Brown, M. B., \& Forsythe, A. B. (1974c). Robust tests for the equality of variances. Journal of the American Statistical Association, 69(346), 364-367. doi: 10.1080/01621459 .1974 .10482955

Browne, M. W. (1984). Asymptotically distribution free methods in the analysis of covariance structures. British Journal of Mathematical and Statistical Psychology, 37(1), 62-83. doi: 10.1111/j.2044-8317.1984.tb00789.x

Chalmers, R. P. (2016). SimDesign: Structure for Organizing Monte Carlo Simulation Designs [software manual]. Retrieved from https://CRAN.R-project.org/package=SimDesign (R package version 1.3)

Cribbie, R. A., Fiksenbaum, L., Wilcox, R. R., \& Keselman, H. J. (2012). Effects of nonnormality on test statistics for one-way independent groups designs. British Journal of Mathematical and Statistical Psychology, 65(1), 56-73. doi: 10.1111/j.2044-8317.2011.02014.x

Curran, P. J., West, S. G, \& Finch, J. F. (1996). The robustness of test statistics to nonnormality and specification error in confirmatory factor analysis. Psychological Methods, 1(1), 16-29. doi: 10.1037/1082-989x.1.1.16

Fan, W., \& Hancock, G. R. (2012). Robust means modeling: An alternative for hypothesis testing of independent means under variance heterogeneity and nonnormality. Journal of Educational and Behavioral Statistics, 37(1), 137-156. doi: 10.3102/1076998610396897 


\section{FURTHER EXPLORING ROBUST MEANS MODELING}

Finch, J. F., West, S. G., \& MacKinnon, D. P. (1997). Effects of sample size and nonnormality on the estimation of mediated effects in latent variable models. Structural Equation Modeling, 4(2), 87-107. doi: 10.1080/10705519709540063

Glass, G. V, Peckham, P. D., \& Sanders, J. R. (1972). Consequences of failure to meet assumptions underlying the fixed effects analysis of variance and covariance. Review of Educational Research, 42(3), 237-288. doi: 10.3102/00346543042003237

Golinski, C., \& Cribbie, R. A. (2009). The expanding role of quantitative methodologists in advancing psychology. Canadian Psychology, 50(2), 83-90. doi: 10.1037/a0015180

Harwell, M. R., Rubinstein, E. N., Hayes, W. S., \& Olds, C. C. (1992). Summarizing Monte Carlo results in methodological research: The one- and two-factor fixed effects ANOVA cases. Journal of Educational Statistics, 17(4), 315-339. doi: 10.3102/10769986017004315

Hoaglin, D.C. (1985). Summarizing shape numerically: The g-and-h distributions. In D.C. Hoaglin, F. Mosteller, and J.W. Tukey (Eds.), Exploring data tables, trends, and shapes, (pp. 461-513). New York: Wiley.

Hoekstra, R., Kiers, H. A. L, \& Johnson, A. (2012). Are assumptions of well-known statistical techniques checked, and why (not)? Frontiers in Psychology, 3, 1-9. doi: 10.3389/ fpsyg.2012.00137

Hu, L., Bentler, P.M., \& Kano, Y. (1992). Can test statistics in covariance structure analysis be trusted? Psychological Bulletin, 112(2), 351-362. doi: 10.1037/0033-2909.112.2.351

James, G. S. (1951). The comparison of several groups of observations when the ratios of population variances are unknown. Biometrika, 38(3/4), 324-329. doi: 10.2307/2332578

Keselman, H. J., Algina, J., Lix, L. M., Wilcox, R. R., \& Deering, K. (2008). A generally robust approach for testing hypotheses and setting confidence intervals for effect sizes. Psychological Methods, 13(2), 110-129. doi: 10.1037/1082-989x.13.2.110

Keselman, H. J., Algina, J., Wilcox, R. R., \& Kowalchuk, R. K. (2000). Testing repeated measures hypotheses when covariance matrices are heterogeneous: Revisiting the robustness of the Welch-James test again. Educational and Psychological Measurement, 60(6), 925-938. doi: 10.1177/00131640021970998

Keselman, H. J., Huberty, C. J., Lix, L. M., Olejnik, S., Cribbie R. A., Donahue, B., Kowalchuk, R. K., . . Levin, J. R. (1998). Statistical practices of educational researchers: An analysis of their ANOVA, MANOVA, and ANCOVA analyses. Review of Educational Research, 68(3), 350-386. doi: 10.3102/00346543068003350

Keselman, H. J., Kowalchuk, R. K., \& Lix, L. M. (1998). Robust nonorthogonal analyses revisited: An update based on trimmed means. Psychometrika, 63(2), 145-163. doi: $10.1007 / \mathrm{bf} 02294772$

Lix, L. M., \& Keselman, H. J. (1998). To trim or not to trim: Tests of location equality under heteroscedasticity and nonnormality. Educational and Psychological Measurement, 58(3), 409-42. doi: 10.1177/0013164498058003004

Lix, L. M., Keselman, J. C., \& Keselman, H. J. (1996). Consequences of assumption violations revisited: A quantitative review of alternatives to the one-way analysis of variance $\mathrm{F}$ test. Review of Educational Research, 66(4), 579-619. doi: 10.3102/00346543066004579

Micceri, T. (1989). The unicorn, the normal curve, and other improbable creatures. Psychological Bulletin, 105(1), 156-166. doi: 10.1037/0033-2909.105.1.156 


\section{ALYSSA COUNSELL ET AL.}

Muthén, B. (1989). Multiple-group structural modeling with non-normal continuous variables. British Journal of Mathematical and Statistical Psychology, 42(1), 55-62. doi: 10.1111/j.2044-8317.1989.tb01114.x

Muthén, B., \& Kaplan, D. (1992). A comparison of some methodologies for the factor analysis of non-normal Likert variables: A note on the size of the model. British Journal of Mathematical and Statistical Psychology, 45(1), 19-30. doi: 10.1111/j.2044-8317.1992. tb00975.x

Olsson, U. H, Foss, T., Troye, S., \& Howell, R. (2000). The performance of ML, GLS, and WLS estimation in structural equation modeling under conditions of misspecification and nonnormality. Structural Equation Modeling: A Multidisciplinary Journal, 7(4), 557-595. doi: 10.1207/s15328007sem0704_3

R Development Core Team. (2014). R: A language and environment for statistical computing [Computer software manual]. Vienna, Austria.

Rosseel, Y. (2012). lavaan: An R package for structural equation modeling. Journal of Statistical Software, 48(2), 1-36. Retrieved from http://www.jstatsoft.org/v48/i02 doi: 10.18637/jss.v048.i02

Satorra, A. (1992). Asymptotic robust inferences in the analysis of mean and covariance structures. Sociological Methodology, 22, 249-278. doi: 10.2307/270998

Satorra, A., \& Bentler, P.M. (1988). Scaling corrections for chi-square statistics in covariance structure analysis. In American Statistical Association 1988. Proceedings of the Business and Economics Sections (pp. 308-313). Alexandria, VA: American Statistical Association.

Satorra, A., \& Bentler, P. M. (2001). A scaled difference chi-square test statistic for moment structure analysis. Psychometrika, 66(4), 507-514. doi: 10.1007/bf02296192

Sorbom, D. (1974). A general method for studying differences in factor means and factor structure between groups. British Journal of Mathematical and Statistical Psychology, 27(2), 229-239. doi: 10.1111/j.2044-8317.1974.tb00543.x

Welch, B. L. (1938). The significance of the difference between two means when the population variances are unequal. Biometrika, 29(3-4), 350-362. doi: 10.1093/biomet/29.3-4.350

Welch, B. L. (1951). On the comparison of several mean values: An alternative approach. Biometrika, 38(3-4), 330-336. doi: 10.1093/biomet/38.3-4.330

Wilcox, R. R. (1988). A new alternative to the ANOVA F and new results on James' second-order method. British Journal of Mathematical and Statistical Psychology, 41(1), 109-117. doi: 10.1111/j.2044-8317.1988.tb00890.x

Wilcox, R. R. (1990a). Comparing the means of two independent groups. Biometrical Journal, 32(7), 771-780. doi: 10.1002/bimj.4710320702

Wilcox, R. R. (1990b). Comparing variances and means when distributions have non-identical shapes. Communications in Statistics-Simulation and Computation, 19(1), 155-173. doi: 10.1080/03610919008812850

Wilcox, R. R. (1995). ANOVA: The practical importance of heteroscedastic methods, using trimmed means versus means, and designing simulation studies. British Journal of Mathematical and Statistical Psychology, 48(1), 99-114. doi: 10.1111/j.2044-8317.1995. tb01052.x

Wilcox, R. R. (2017). Introduction to robust estimation and hypothesis testing, 4th ed. San Diego, CA: Academic Press. 


\section{FURTHER EXPLORING ROBUST MEANS MODELING}

Wilcox, R. R., Keselman, H. J., Muska, J., \& Cribbie, R. (2000). Repeated measures ANOVA: Some new results on comparing trimmed means and means. British Journal of Mathematical and Statistical Psychology, 53(1), 69-82. doi: 10.1348/000711000159187

Yuan, K. H., \& Bentler, P.M. (1997). Mean and covariance structure analysis: Theoretical and practical improvements. Journal of the American Statistical Association, 92(438), 767-774. doi: 10.1080/01621459.1997.10474029

Yuan, K. H., \& Bentler, P. M. (1999). F tests for mean and covariance structure analysis. Journal of Educational and Behavioral Statistics, 24(3), 225-243. doi: 10.3102/ 10769986024003225 